\title{
HST Observations of Young Stellar Clusters in Nearby Galaxies
}

\author{
Søren S. Larsen \\ European Southern Observatory, Karl-Schwarzschild-Str. 2, D-85748 \\ Garching bei München, Germany
}

\begin{abstract}
The HST data archive contains images of several nearby spirals, suitable for detailed studies of the properties of individual star clusters and their surroundings. By combining structural information derived from HST images with ground-based photometry, it is possible to study cluster properties as a function of age and mass. While both the core and effective radii of young clusters correlate with mass, the slopes of these relations are shallower than for a constant-density relation, implying that the mean density increases with cluster mass. This must be accounted for in theories for cluster formation.
\end{abstract}

\section{Introduction}

While the rich cluster systems in galaxy mergers and starbursts have been studied intensively over the past decade, it is sometimes overlooked that even some "normal", undisturbed spiral galaxies can form highly luminous, young star clusters (Larsen \& Richtler 1999; Paper I). Within a distance of $10 \mathrm{Mpc}$, there are several examples of spirals with rich cluster systems which have been imaged with HST/WFPC2, allowing the properties and environments of individual star clusters to be studied in great detail. By combining the HST images with ground-based photometry, it is possible to look for trends in structural parameters with cluster age and mass, providing potentially valuable clues to the physics of cluster formation and subsequent dynamical evolution.

\section{Cluster structure versus mass and age}

We have recently scanned the HST archive for images of galaxies included in the original survey from Paper I. A total of 17 galaxies were found to have adequate HST data (Larsen 2004). The constraint that the clusters have to be visible on ground-based images is not as severe as it might appear at first, since a high $\mathrm{S} / \mathrm{N}$ in the HST images is required anyway to measure accurate structural parameters. By fitting "Moffat" models of the form $P(r) \propto\left[1+\left(r / r_{c}\right)^{2}\right]^{-\alpha}$, it is possible to constrain both the core radius $r_{c}$ and the shape of the luminosity profile at large radii, parameterized by the slope $\alpha$. While these profiles have no physical motivation, they have been shown to fit LMC clusters well (Elson et al. 1987). For $\alpha=1$, they are identical to a King (1962) model with infinite concentration parameter.

The left panel in Fig. 1 shows the distribution of envelope slopes for clusters in four age bins. There is a clear tendency for many of the youngest clusters to have extended outer envelopes. This may reflect the density structure of the 

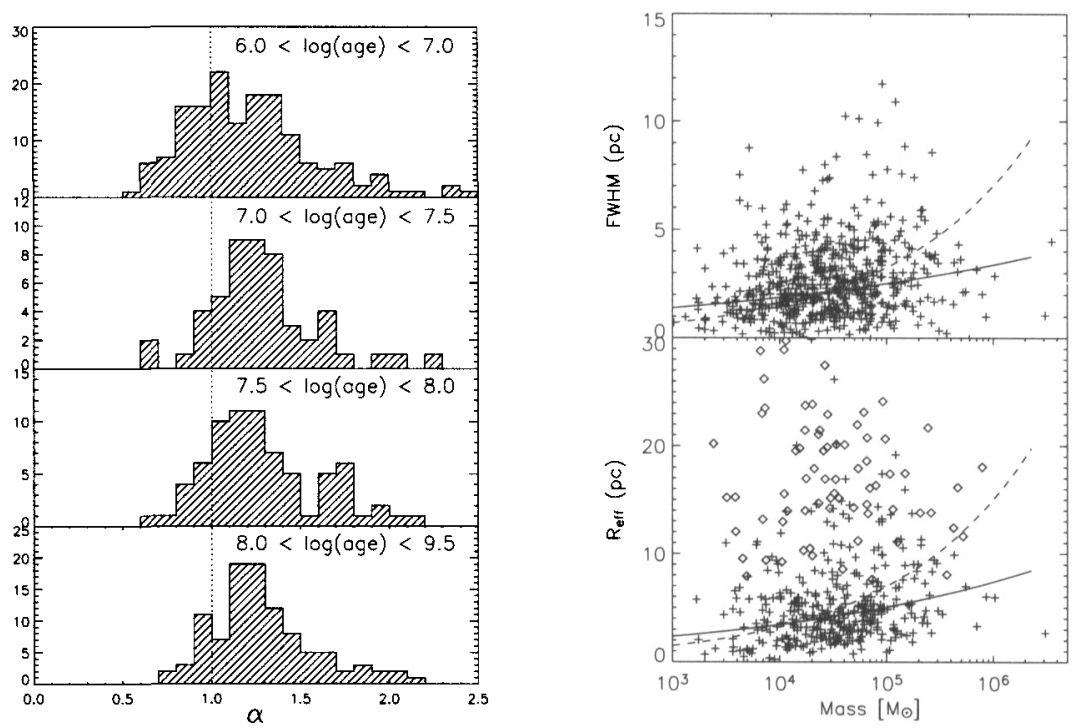

Figure 1. Left: Distribution of envelope slopes for clusters in four age bins. Right: FWHM and half-light radius versus mass.

parent molecular cloud cores, or be a hint that some of these clusters are unbound and expanding. Older clusters gradually evolve towards a King-like profile. In the right-hand panel, the FWHM and half-light radii are shown versus cluster mass. In each panel, the constant-density relation (size $\propto M^{1 / 3}$ ) is shown with a dashed line, while the solid lines indicate a fit to the data. For the half-light radii, only clusters with $1<\alpha<5$ were included in the fit (clusters with $\alpha<1$ have poorly defined half-light radii and are shown with diamond symbols). Although both relations show substantial scatter, the fits are significantly shallower than for the constant-density relation. This conclusion is robust to selection effects, which would be expected to produce a bias against extended, low-mass objects.

The main limitation in this study is the difficulty of detecting low-mass clusters older than a few times $10^{7}$ years. This situation is expected to improve when new ACS data become available.

\section{References}

Elson, R. A. W., Fall, S. M. \& Freeman, K. C. 1987, ApJ, 323, 54

King, I. R. 1962, AJ, 67, 471

Larsen, S. S. \& Richtler, T. 1999, A\&A, 345, 59

Larsen, S. S. 2004, A\&A, 416, 537 